\title{
IMPACTOS POTENCIAIS DA AGRICULTURA DE PRECISÃO SOBRE A ECONOMIA BRASILEIRA $^{1}$
}

\author{
Cinthia Cabral da Costa ${ }^{2}$ \\ Joaquim José Martins Guilhoto ${ }^{3}$
}

Resumo: O objetivo deste estudo foi estimar o impacto potencial da adoção das principais técnicas de agricultura de precisão na agricultura brasileira sobre fatores econômicos do país. Para isso, inicialmente realizou-se uma revisão bibliográfica dos principais resultados advindos das técnicas de agricultura de precisão. Para simular os impactos destas técnicas na agricultura brasileira, foi considerado o potencial da atividade agrícola que pode ser beneficiada por estas tecnologias para os produtos cana-de-açúcar, milho e soja. Em seguida, os impactos sobre toda a economia foram analisados utilizando a matriz insumo-produto do país. Observou-se que um cenário de aumento de $10 \%$ na produtividade destas culturas, com ou sem redução ou aumento de fertilizantes, poderia aumentar em cerca de $\mathrm{R} \$ 11$ bilhões o PIB da economia brasileira e gerar mais de 450 mil empregos. Em contrapartida, um cenário em que ocorre apenas redução no uso de fertilizantes não mostrou impactos econômicos favoráveis, considerando toda a economia do país, embora os benefícios ambientais possam sempre estar presentes.

Palavras-chave: emprego, PIB, agricultura de precisão, produtividade, insumoproduto.

1 Recebido em: 11/09/2012; Aceito em: 29/01/2013.

2 Pesquisadora. Embrapa Instrumentação. Rua XV de novembro, 1452. São Carlos, SP 13560-970. E-mail: cinthia-costa@bol.com.br.

3 Professor titular. Departamento de Economia, FEA/USP. Av. Prof. Luciano Gualberto, 908. FEA2. Cidade Universitária. São Paulo, SP 05508-010.

E-mail: guilhoto@usp.br. 


\begin{abstract}
The aim of this study is to measure the potential impact in the Brazilian economy due to an increase in the use precision agriculture. Initially, it makes a literature review about the main advantages of using precision agriculture as compared to the tradition techniques. To estimate the potential impacts of the precision agriculture, first, it is defined the cultures which would most benefit from it, after which, and by using input-output techniques, the impacts over GDP and employment were estimated. The cultures analyzed were: sugarcane, corn and soybeans. It was observed that a scenario of a $10 \%$ increase in productivity of all these three crops, with or without reduction or increase in fertilizer, would result in an increased about R $\$ 11$ billion in Brazilian GDP and more than 450.000 new jobs. A scenario in which only the use of fertilizers is reduced did not show any significant economic impacts, however, it should be stressed that this work did not take into consideration the positive environmental impacts which would result from the reduction in the use of fertilizers.
\end{abstract}

Keywords: employment, GDP, precision agriculture, productivity, input-output.

\title{
1. Introdução
}

O presente estudo buscou estimar o impacto potencial sobre fatores econômicos do país da adoção das principais técnicas de agricultura de precisão na agricultura brasileira. A lucratividade ou não das técnicas e sua mensuração não foram objetivo desta análise. Entretanto, foram consideradas, como potenciais para uso da agricultura de precisão, as condições identificadas em outros estudos como aquelas favoráveis à obtenção de lucro - área com significativa variabilidade.

Conforme descrito por Plant (2001), a agricultura de precisão ou manejo sítio-específico é o manejo de lavouras agrícolas ou pecuárias em escalas espaciais menores do que aquelas normalmente adotadas para toda a lavoura. Segundo este autor, muitos agricultores irão decidir por adotar as práticas do manejo sítio-específico e começar a influenciar e aprender o uso da tecnologia somente quando eles forem convencidos de que o tempo e o dinheiro despendidos são justificados pela melhoria na produtividade ou na redução de custos ou de riscos. Neste sentido, Plant cita Miller et al. (1999), que listam três critérios necessários para justificar a introdução destas práticas. São eles: (i) que haja uma significante variabilidade 
espacial na fazenda em fatores que influenciam a produtividade; (ii) que as causas desta variabilidade possam ser identificadas e mensuradas; e (iii) que a informação destas medidas possa ser utilizada para modificar as práticas para aumentar o lucro ou reduzir os impactos ambientais. Desta forma, entende-se que os benefícios do uso da agricultura de precisão devem ser analisados caso a caso.

Para um levantamento sobre quais técnicas da agricultura de precisão são mais empregadas, utilizou-se o estudo de Whipker \& Akridge (2009). Os autores mostraram que, para uma amostra dos produtores rurais nos EUA, no ano de 2009 (em que 85\% deles utilizaram alguma técnica de agricultura de precisão), 62,6\% utilizaram os métodos de agricultura de precisão para aplicação de fertilizantes e 55,5\% os utilizaram para aplicação de pesticidas. Já no Brasil, o único estudo com levantamentos desta natureza é o de Silva et al. (2011). Entretanto, estes autores analisaram apenas uma amostra, referente ao ano de 2008, de usinas produtoras de cana-de-açúcar no país. Neste estudo, os autores encontraram que, das 49 usinas que adotaram a técnica (56\% da amostra analisada), apenas $29 \%$ adotaram a tecnologia de aplicação a taxa variada. A grande maioria utilizou apenas as imagens de satélite. Ou seja, sabe-se que o uso maciço da agricultura de precisão não é uma realidade no país. Assim, outra pressuposição adotada neste estudo é a de que os benéficos potenciais da agricultura de precisão ainda não eram observados no Brasil para o ano de 2006 (ano utilizado no presente estudo). Além disto, pelos resultados de pesquisas americanas, a técnica de agricultura de precisão para aplicação de fertilizantes foi considerada a mais usual.

O principal benefício da agricultura de precisão é o uso eficiente dos insumos agrícolas. Como resultado, pode-se ter um ou mais dos seguintes efeitos positivos: (i) redução nos custos pela diminuição no uso dos insumos; (ii) redução na poluição da água e do ambiente; e (iii) aumento da produtividade agrícola pela aplicação mais eficiente dos insumos. Contudo, os impactos econômicos podem ser identificados na sociedade como resultado dos efeitos relacionados nos itens (i) e (iii). Portanto, o presente estudo buscou traçar um cenário geral para identificar os 
impactos econômicos com base nos benefícios originados pelo uso da técnica da agricultura de precisão na agricultura brasileira, considerando seus efeitos sobre a redução no uso de insumos e/ou aumento na produtividade. Ou seja, para o agricultor, o uso desta tecnologia pode se traduzir em aumento de renda, mas qual o efeito sobre toda economia? Há algum crescimento econômico proveniente deste resultado?

Para atingir o objetivo deste estudo, foi realizada uma revisão de literatura sobre os efeitos das práticas de agricultura de precisão sobre o uso de insumos, a produtividade e a contaminação ambiental. Esta revisão é importante na identificação dos choques que serão aplicados para obtenção dos resultados estimados neste trabalho (item 2). O item 3 apresenta a descrição do método utilizado no estudo e no item 4 são apresentados os cenários analisados e os impactos resultantes sobre a economia pela utilização da agricultura de precisão. O item 5 apresenta as conclusões do trabalho.

\section{Revisão de literatura: efeitos das práticas de agricultura de precisão}

Primeiramente, é importante destacar que, apesar de o foco deste estudo ser o impacto da agricultura de precisão sobre a agricultura e a economia do Brasil, a maior parte das revisões bibliográficas feitas não se restringe apenas à análise das condições brasileiras, em função da existência de, relativamente, muito mais estudos feitos em outros países. Na Tabela 1 , são descritos os resultados de alguns estudos que estimam impactos sobre o uso dos insumos e/ou produtividade. É importante destacar que os cinco últimos trabalhos são específicos para as condições brasileiras. Conforme observado nos trabalhos descritos na Tabela 1, há diversidade tanto em relação ao método utilizado quanto aos resultados. Em relação ao método de aplicação de precisão, tem-se a aplicação de fertilizantes, agrotóxicos e a irrigação. Mais comumente na literatura, são encontrados trabalhos sobre aplicação de precisão para fertilizantes nitrogenados. Isto se justifica pelos altos índices de contaminação de rios americanos com 
nitrato e que, conforme descrito por Raun \& Johnson (1999), a eficiência no uso do nitrogênio é de apenas 33\%. Ou seja, os 67\% restantes são lixiviados e contaminam o ambiente. Alguns trabalhos apresentam ainda o uso da agricultura de precisão para outras atividades, como a colheita (TOZER \& ISBISTER, 2007), e para avaliação de outras características, como a qualidade do produto (LONG et al., 2000).

A análise dos resultados apresentados na Tabela 1 pode ser feita por cultura ou por tipo de insumo aplicado pelo método de precisão. Neste trabalho, foi adotada esta última forma. Assim, verificou-se que a redução no uso do nitrogênio, utilizando aplicação variada, foi entre 18 e 50\%, sem referências significativas para a produtividade em culturas como cana, milho e trigo (RUFFO et al., 2008; BONFIL et al., 2008; KOCH et al., 2003; MCKINION et al., 2001; GRIEPENTROG \& KYHN, 2000; AHMAD et al., 1997; STONE et al., 1996). Trabalhos conduzidos nas condições nacionais, por sua vez, indicaram redução de apenas $2 \%$ de nitrogênio no café e aumento de $6 \%$ no arroz. Entretanto, para estes últimos trabalhos, houve, conjuntamente, variações em fósforo e potássio. Assim, na cultura do café, houve redução de $2 \%$ no nitrogênio e $1 \%$ no fósforo, mas houve incremento de $7 \%$ em potássio, e a produtividade aumentou em 2\% (FAULIN et al., 2010). Já na cultura do arroz, todos os insumos aumentaram com a aplicação variada (ACOSTA et al., 2010). A produtividade, por sua vez, aumentou em 3\%, e os autores mostraram que o aumento de receitas proveniente deste aumento de produtividade foi superior ao aumento dos custos dos insumos.

Ainda em relação à aplicação diferenciada de nutrientes, Molin et al. (2010) mediram a resposta da aplicação à taxa variável de fósforo e potássio. A área que recebeu doses variadas apresentou produtividade $34 \%$ maior, de $1677,9 \mathrm{~kg} /$ ha para $2556,6 \mathrm{~kg} / \mathrm{ha}$, se comparada com a área que recebeu adubação em doses fixas. Houve também redução de $23 \%$ no consumo de fósforo, que passou de 417 para $319 \mathrm{~kg} / \mathrm{ha}$, e aumento de $13 \%$ no consumo de potássio, que passou de 334 para $387 \mathrm{~kg} / \mathrm{ha}$. Trabalhos avaliando consumo de calcário indicaram que métodos de agricultura de precisão reduziram o uso deste insumo entre 27-30\% (LOPES \& 
MOLIN, 2010; BARBIERI et al., 2008). No trabalho de Menegatti et al. (2006), apesar de não ser explicitada a variação no uso de calcário, houve referência em relação ao aumento de $9 \%$ da produtividade da cana-de-açúcar pelo uso da agricultura de precisão.

Tabela 1. Estudos sobre o impacto da agricultura de precisão (aplicação diferenciada de insumos) sobre o consumo dos insumos agrícolas e sobre a produtividade em relação ao uso uniforme dos insumos

\begin{tabular}{|c|c|c|c|c|}
\hline Fonte & $\begin{array}{c}\text { Produto } \\
\text { analisado }\end{array}$ & $\begin{array}{c}\text { Insumo } \\
\text { avaliado }\end{array}$ & $\begin{array}{l}\text { Variação no uso do } \\
\text { insumo }\end{array}$ & $\begin{array}{c}\text { Variação na } \\
\text { Produtividade }\end{array}$ \\
\hline $\begin{array}{c}\text { Griepentrog e Kyhn } \\
(2000)\end{array}$ & $\begin{array}{l}\text { Trigo e } \\
\text { barley }\end{array}$ & $\mathrm{N}$ & $-36 \%$ & - \\
\hline Mckinion et al. (2001) & Algodão & N e água & $\begin{array}{c}+2,6 \mathrm{~cm} / \mathrm{ha} \text { de água; }-35 \\
\mathrm{~kg} / \mathrm{ha} \mathrm{de} \mathrm{N}\end{array}$ & $+322 \mathrm{~kg} / \mathrm{ha}$ \\
\hline Ahmad et al. (1997) & Milho & N e água & $\begin{array}{c}-18,4 \% \text { para } \mathrm{N} ;-5,9 \% \mathrm{de} \\
\text { água }\end{array}$ & $-4,6 \%$ \\
\hline Stone et al. (1996) & Trigo & $\mathrm{N}$ & até $-50 \%$ & \\
\hline Koch et al. (2003) & Milho & $\mathrm{N}$ & $-46 \%$ & \\
\hline Menegatti et al. (2006) & $\begin{array}{l}\text { Cana-de- } \\
\text { açúcar }\end{array}$ & $\begin{array}{l}\text { Calcário, } \\
\text { gesso e P }\end{array}$ & $-15 \%$ custos & $+9 \%$ \\
\hline Ruffo et al. (2008) & $\begin{array}{l}\text { Etanol de } \\
\text { milho }\end{array}$ & $\mathrm{N}$ & $-36 \%$ & $+0,7$ \\
\hline Hedley \& Yule (2008) & $\begin{array}{l}\text { Milho e } \\
\text { pastagem }\end{array}$ & $\begin{array}{l}\text { Água de } \\
\text { irrigação }\end{array}$ & $\begin{array}{c}-26,3 \% \text { no milho; }-21,8 \% \\
\text { na pastagem }\end{array}$ & \\
\hline Bonfil et al. (2008) & trigo & $\mathrm{N}$ & $+73 \mathrm{~kg} / \mathrm{ha}$ & $+8 \%$ \\
\hline Molin et al. (2010) & Café & $\mathrm{P}$ e $\mathrm{K}$ & $-13 \%$ de $\mathrm{P} ;+13 \%$ de $\mathrm{K}$ & $+34 \%$ \\
\hline Barbieri et al. (2008) & $\begin{array}{l}\text { Cana-de- } \\
\text { açúcar }\end{array}$ & $\begin{array}{c}\text { Calcário } \\
\text { dolomític } \\
\quad \text { o }\end{array}$ & $-30 \%$ & - \\
\hline Lopes \& Molin (2010) & Citrus & $\begin{array}{l}\mathrm{P}, \mathrm{Ke} \\
\text { calcário }\end{array}$ & $\begin{array}{c}-27 \% \text { de calcário; }-53,5 \% \\
\text { de } \mathrm{P} ; \\
-47,5 \% \text { de } \mathrm{K}\end{array}$ & - \\
\hline Faulin et al. (2010) & Café & $\mathrm{N}, \mathrm{P}$ e $\mathrm{K}$ & $\begin{array}{c}-2 \% \text { de } \mathrm{N} ;-1 \% \text { de } \mathrm{Pe} \\
+7 \% \text { de } \mathrm{K}\end{array}$ & $+2 \%$ \\
\hline Acosta et al. (2010) & Arroz & $\mathrm{N}, \mathrm{P}$ e K & $\begin{array}{c}+6 \% \text { de } \mathrm{N} ;+75 \% \text { de } \mathrm{Pe} \\
+80 \% \text { de } \mathrm{K}\end{array}$ & $+3 \%$ \\
\hline
\end{tabular}

Nota: $\mathrm{P}$ indica fósforo; $\mathrm{K}$ representa o potássio, e $\mathrm{N}$ o nitrogênio.

Embora em menor número do que os trabalhos que avaliam impacto sobre fertilizantes, também há indicações de que métodos de agricultura de precisão podem reduzir o consumo de água de irrigação em mais de $20 \%$ para milho e pastagem (HEDLEY \& YULE, 2008). Outros trabalhos 
mostraram redução em uma proporção menor para milho e algodão (AHMAD et al., 1997; MCKINION et al., 2001).

A conclusão que pode ser retirada desta revisão é que não há um padrão para alterações no uso de insumos e da produtividade, pois foi verificada grande diversidade de resultados, considerando a aplicação diferenciada de insumos em relação à aplicação uniforme. E também foi verificado que a redução no consumo de fertilizantes foi o alvo principal dos impactos no uso de técnicas de agricultura de precisão.

Apesar dos benefícios citados, os trabalhos que apresentam resultados inconsistentes em termos dos benefícios financeiros das técnicas de agricultura de precisão para o produtor não são raros. Isto não significa que a técnica não seja ou não possa se tornar eficiente. Segundo Tekin (2010), o principal fator para o sucesso na implementação da agricultura de precisão é o grau de variabilidade, em que maiores graus facilitam a implementação. Adicionalmente a esta ideia, Molin (1997) descreve que normalmente existe uma variabilidade muito grande nas propriedades do solo e que esta variabilidade cresce com o tamanho da lavoura. Áreas com fertilidade muito baixa ou muito alta tendem também a ser mais homogêneas e, portanto, a responder menos às técnicas de agricultura de precisão. Corroborando esta ideia, English, Roberts e Larson (2000) descrevem que a maior diversidade dos solos está diretamente correlacionada com a efetividade no uso da agricultura de precisão.

Adicionalmente, Plant (2001) descreve que uma das críticas à agricultura de precisão é que gera mais benefícios para os grandes produtores agrícolas. Segundo este autor, um argumento comum é que grandes propriedades são econômica e agronomicamente ineficientes porque não levam em conta a variabilidade inerente. Entretanto, isto não é a realidade em países como o Brasil, onde os produtores menores são, também, menos capitalizados para investir em insumos que aumentam a produtividade das suas lavouras. Mas esta é mais uma indicação de que os efeitos benéficos destas práticas são mais restritos a grandes áreas de lavouras. 
Já no que se refere aos impactos ambientais do uso de técnicas de precisão, a Tabela 2 descreve alguns estudos (há muitos outros, principalmente na década de 1990, que apresentam resultados semelhantes) indicando alguma convergência de resultados. Entre os nutrientes, o impacto ambiental mais destacado se refere ao nitrogênio, uma vez que é facilmente lixiviado no solo e contamina os lençóis freáticos. Entretanto, em relação ao impacto ambiental, não foram encontradas referências de estudos no país.

Os estudos descritos na Tabela 2 mostraram uma redução no nitrogênio lixiviado entre 13 e 50\%. Já a redução na contaminação ambiental resultante da aplicação precisa de agrotóxicos foi, para todos os estudos relatados, maior do que $50 \%$, o que mostra o grande potencial de redução desta contaminação pela adoção da agricultura de precisão.

Tabela 2. Estudos sobre o impacto da agricultura de precisão (aplicação diferenciada de insumos) sobre o ambiente em relação ao uso uniforme dos insumos

\begin{tabular}{|c|c|c|c|}
\hline Fonte & $\begin{array}{c}\text { Produto } \\
\text { Analisado }\end{array}$ & $\begin{array}{c}\text { Insumo } \\
\text { avaliado }\end{array}$ & $\begin{array}{l}\text { Redução na lixiviação de } \\
\text { nutrientes } \\
\text { ou contaminação ambiental }\end{array}$ \\
\hline Du et al. (2008) & Citros & Pesticida & $92 \%$ \\
\hline $\begin{array}{l}\text { Delgado e Bausch } \\
\text { (2005) }\end{array}$ & Milho & $\mathrm{N}$ & $47 \%$ \\
\hline Delgado et al. (2005) & & $\mathrm{N}$ & $25 \%$ \\
\hline Roberts et al. (2002) & Milho & $\mathrm{N}$ & 2 a 4 libras/acre \\
\hline Roberts et al. (2001) & Milho & $\mathrm{N}$ & 2,24 a $4,48 \mathrm{~kg} / \mathrm{ha}$ \\
\hline $\begin{array}{l}\text { Timmermann et al. } \\
\text { (2001) }\end{array}$ & $\begin{array}{l}\text { Trigo, milho, } \\
\text { beterraba, } \\
\text { cevada }\end{array}$ & herbicida & $-54 \%$ \\
\hline $\begin{array}{c}\text { Thrikawala et al. } \\
\text { (1999) }\end{array}$ & milho & $\mathrm{N}$ & $\begin{array}{c}-13 \%(-4,2 \% \text { em áreas de alta } \\
\text { fertilidade e }-36,3 \% \text { em áreas de } \\
\text { baixa fertilidade })\end{array}$ \\
\hline Tian et al. (1999) & milho & herbicida & $-42 \%$ \\
\hline $\begin{array}{l}\text { Carpentier et al. } \\
(1998)\end{array}$ & pastagem & $\mathrm{N}$ & $-40 \%$ \\
\hline Ahmad et al. (1997) & Milho & $\mathrm{N}$ e água & $-15,7 \%$ \\
\hline Larson et al. (1997) & milho & $\mathrm{N}$ & $-50 \%$ (de 60 para $29 \mathrm{~kg} / \mathrm{ha}$ ) \\
\hline $\begin{array}{l}\text { Nordmeyer et al. } \\
\text { (1997) }\end{array}$ & cereais & herbicida & $-47-80 \%$ \\
\hline
\end{tabular}

Nota: P indica fósforo; K representa o potássio e N, o nitrogênio. 
Uma característica a considerar em relação à mensuração dos impactos ambientais da agricultura de precisão é que eles sempre ocorrerão, uma vez que há redução na perda de insumos para o ambiente. Neste sentido, Delgado \& Berry (2008) e Delgado et al. (2010), considerando os incontestáveis benefícios ambientais destas técnicas, utilizaram um conceito diferente para o uso das tecnologias utilizadas na Agricultura de Precisão, chamando-as de "Conservação de Precisão" (CP). Neste conceito, os autores expandem aquele definido pela "Agricultura de Precisão" (AP) para uma perspectiva mais interconectada com o ambiente: enquanto a AP considera apenas a área de lavoura, a CP conecta campos agrícolas diferentes, pastagens, áreas vizinhas, matas ciliares, florestas e corpos d'água. Estes autores propõem que este sistema será a chave da ciência que irá contribuir para a sustentabilidade de um sistema agrícola intensivo no futuro, pois ajudará a analisar as relações espaciais e temporais para melhor entendimento dos sistemas agrícolas e naturais. Neste ponto cabe uma reflexão: não será o benefício das tecnologias da agricultura de precisão mais ambiental do que econômico?

Enquanto esta resposta não esteja pronta, os defensores do uso da agricultura de precisão defendem lucros derivados deste sistema, seja por redução de insumo e/ou por aumento de produtividade. Assim, o presente estudo utilizou tais choques descritos pela Agricultura de Precisão para responder quais os impactos para a economia brasileira derivados dos choques provocados sobre a produtividade e uso de insumos.

\section{Material e métodos}

Inicialmente foi feita uma análise sobre as condições da agricultura brasileira e do potencial de adoção destas tecnologias, segundo o conceito de AP. Neste conceito, a adoção deve ser feita em áreas de lavouras com grande variabilidade. Para isto, foram analisadas as informações do último censo agropecuário do país, do ano de 2006. Com base nos resultados da revisão feita anteriormente (item 2) e na análise da produção agropecuária de 2006, foram traçados os panoramas e elaborados os 
cenários para identificar os impactos da adoção da agricultura de precisão na economia do país. Nesta etapa, foram identificados os principais produtos agrícolas que se beneficiariam desta técnica.

Considerando os produtos selecionados para análise, eles foram desagregados em setores específicos nas Tabelas de Recursos e Usos das Contas Nacionais do ano de 2006. A desagregação foi feita a partir do setor "Agricultura, silvicultura e exploração florestal". A partir das tabelas de Recursos e Usos, foi estimada a matriz insumo-produto conforme metodologia descrita em Guilhoto \& Sesso (2005). A seguir, é descrita a análise feita na segunda etapa do trabalho, utilizando esta matriz insumo-produto estimada.

A segunda etapa do trabalho teve como objetivo estimar os impactos de todos os encadeamentos provocados na economia pelos cenários elaborados. Para isto, foi utilizado o instrumental de avaliação da matriz insumo-produto (MIP). Esta análise parte de um choque de aumento de demanda em setores da economia que causam um aumento na produção, considerando todos os encadeamentos entre tais setores (MILLER \& BLAIR, 2009).

A análise da matriz de insumo-produto do país representa as relações de demanda intermediária e é descrita na forma matricial como " $A$ ". Assim, pode-se descrever o valor da produção de uma economia na equação (1):

$A X+Y=X$

em que as matrizes " $X$ " e " $Y$ " são, respectivamente, as matrizes de valor bruto da produção e de demanda final. De acordo com Miller \& Blair (2009), os resultados sobre o valor da produção da economia são obtidos conforme descrito na equação (2):

$X=(I-A)^{-1} * Y$

em que $(I-A)^{-1}$ é a matriz de coeficientes técnicos de insumo diretos e indiretos, conhecida como a matriz inversa de Leontief. 
É a partir da matriz inversa de Leontief que se obtêm os chamados multiplicadores de impactos de um choque de demanda final (choque na variável Y) na economia. Tais multiplicadores de impactos são os efeitos diretos do choque proposto, assim como os indiretos (a jusante da cadeia produtiva). Além dos impactos diretos e indiretos, podem também ser obtidos os impactos de efeito renda, que correspondem ao aumento de demanda das famílias resultante dos impactos diretos e indiretos. Para obter este efeito adicional na economia, a matriz inversa de Leontief é obtida a partir de uma matriz $\bar{A}$ de coeficientes técnicos, em que se considera a endogeneização no consumo das famílias, de modo que o multiplicador setorial é definido a partir da matriz .

Analogamente ao descrito anteriormente, para estimar o valor de produção da economia $(\bar{X})$, que é acionado para atender a uma variação exógena na demanda final $(Y)$, tem-se:

$$
\bar{X}=(I-\bar{A})^{-1} * Y
$$

Utilizando o multiplicador de impacto $(I-\bar{A})^{-1}$, foram então calculados os impactos na economia sobre número de empregos $\left(Z_{E}\right)$, valor das remunerações $\left(Z_{R}\right)$, valor das importações $\left(Z_{M}\right)$ e produto interno bruto - PIB $\left(Z_{P I B}\right)$. Para isto, os choques na produção estimados na equação (3) foram multiplicados pelos coeficientes das variáveis emprego $\left(C_{E}\right)$, remunerações $\left(C_{R}\right)$, importação $\left(C_{M}\right)$ e PIB $\left(C_{P I B}\right)$. Todos estes coeficientes foram obtidos também de dados identificados na matriz insumo-produto. O coeficiente de cada uma destas variáveis (emprego, remuneração, importação e PIB) é obtido dividindo, para cada setor da MIP, o valor de cada uma destas informações pelo seu valor da produção.

Apesar de a análise convencional da matriz insumo-produto ser no sentido de impacto de aumento na demanda final (variável $Y$ ), neste trabalho a análise foi feita considerando outros choques na matriz insumo-produto, os quais simulam o efeito da agricultura de precisão. 
São eles: (a) aumento de produtividade das culturas; (b) redução no uso de fertilizantes químicos; (c) aumento de produtividade com redução no uso de fertilizantes químicos; e (d) aumento de produtividade com aumento no uso de fertilizantes químicos. A Tabela 3 descreve quais matrizes foram alteradas para estimação dos resultados em cada um dos cenários. Assim, as variáveis $Y_{l}, A_{1}$ e $P I B_{l}$ com índice "1", nesta tabela, são aquelas estimadas a partir da realidade para o ano de 2006. $A_{l}$ e $P I B_{l}$ foram calculadas a partir das Contas Nacionais e $Y_{1}$, pela demanda final estimada a partir do IBGE (2012), que corresponde à produção que pode ser afetada pelo uso da agricultura de precisão analisada nesse estudo.

Tabela 3. Matrizes utilizadas para estimativa dos impactos que simulam os efeitos da agricultura de precisão

Cenário Base

Cenário Cenário $\quad$ Cenário

Cenário

(a)

(b)

(c)

(d)

\begin{tabular}{lccccc}
\hline $\begin{array}{l}\text { Vetor da demanda } \\
\text { final }(Y)\end{array}$ & $Y_{1}$ & $Y_{2}$ & $Y_{1}$ & $Y_{2}$ & $Y_{2}$ \\
\hline $\begin{array}{l}\text { Matriz de } \\
\text { coeficientes } \\
\text { técnicos }(A)\end{array}$ & $A_{1}$ & $A_{2}$ & $A_{3}$ & $A_{4}$ & $A_{5}$ \\
\hline $\begin{array}{l}\text { Vetor de } \\
\text { coeficientes do PIB } \\
\left(C_{P I B}\right) .\end{array}$ & $C_{P I B, 1}$ & $C_{P I B, 2}$ & $C_{P I B, 3}$ & $C_{P I B, 4}$ & $C_{P I B, 5}$ \\
\hline
\end{tabular}

Nota: Descrição dos cenários: (a) aumento de produtividade das culturas; (b) redução no uso de fertilizantes químicos; (c) aumento de produtividade com redução no uso de fertilizantes químicos e (d) aumento de produtividade com aumento no uso de fertilizantes químicos.

Para estimar o impacto de aumento na produtividade das culturas selecionadas, alterou-se o vetor da demanda final $\left(Y_{2}\right)$ de maneira que os setores analisados tivessem uma demanda superior, correspondente ao aumento de produtividade estimada. Simultaneamente, todos os coeficientes técnicos daqueles setores foram reduzidos na mesma proporção (obtendo a matriz $A_{2}$ ), pois o aumento na produção foi 
decorrente de aumento na produtividade e não de uso de insumo. Já para estimar a redução no uso de fertilizante, foi alterada a matriz de coeficientes técnicos de maneira que fosse necessário menos do fertilizante para alcançar o mesmo valor da produção (obtendo a matriz $\left.A_{3}\right)$. Nos cenários que misturam aumento de produtividade com redução ou aumento de fertilizante, cenários $\mathrm{c} \mathrm{e} \mathrm{d}$, respectivamente, foram adicionados à matriz de coeficiente técnico $A_{2}$, redução do coeficiente do uso de fertilizante, obtendo a matriz $A_{4}$ (cenário c), e o aumento do coeficiente do uso de fertilizante, obtendo a matriz $A_{5}$ (para o cenário d). A variável referente ao coeficiente do $\operatorname{PIB}\left(C_{P I B}\right)$ foi também alterada porque, uma vez alterados os valores dos coeficientes técnicos de produção, esta variável precisa também ser reestimada de maneira a contrabalançar os dados e fazer o fechamento da MIP. Na prática, funciona da seguinte maneira: se houver redução no uso de fertilizante de uma cultura, o valor do lucro do produtor irá aumentar no mesmo montante, considerando o mesmo valor de produção. Uma vez que o lucro do produtor está refletido no Valor Adicionado (ou PIB), esta variável deve ser alterada.

Uma vez obtidas as matrizes definidas na Tabela 3, os resultados para cada um dos quatro cenários descritos anteriormente foram obtidos a partir das equações descritas na Tabela 4. 
Tabela 4. Equações com as respectivas matrizes utilizadas na obtenção das estimativas dos impactos que simulam os efeitos da agricultura de precisão

\begin{tabular}{lcc}
\hline & $\begin{array}{c}\text { Impacto no Valor da } \\
\text { Produção }(X)\end{array}$ & $\begin{array}{c}\text { Impacto nas demais } \\
\text { variáveis }\left(Z_{l}\right)^{*}\end{array}$ \\
\hline $\begin{array}{l}\text { Cenário Base } \\
X_{B A S E}=\left(I-\overline{A_{1}}\right) * Y_{1}\end{array}$ & $\begin{array}{c}Z_{i, B A S E}=X_{B A S E} * C_{i} \\
\text { se } i=\mathrm{PIB}=C_{P I B, I}\end{array}$ \\
$\begin{array}{l}\text { (a) aumento de produtividade das culturas } \\
\text { (b) redução no uso de fertilizantes químicos }\end{array}$ & $X_{a}=\left(I-\overline{A_{2}}\right) * Y_{2}$ & $\begin{array}{c}Z_{i, a}=X_{a} * C_{i} \\
\text { se } i=\mathrm{PIB}=C_{P I B, 2}\end{array}$ \\
\hline $\begin{array}{l}\text { (c) aumento de produtividade com redução } \\
\text { no uso de fertilizantes químicos }\end{array}$ & $X_{c}=\left(I-\overline{A_{3}}\right) * Y_{1}$ & se $i=\mathrm{PIB}=C_{P I B, 3}$ \\
\hline
\end{tabular}

0

Nota: $* Z$, sendo $i=$ " $E$ " para emprego; " $R$ " para remuneração; " $M$ " para importação e " $P I B$ " para produto interno bruto.

A diferença entre os impactos na economia advindos dos resultados obtidos nos cenários descritos em (a), (b), (c) e (d) em relação ao Cenário Base correspondeu aos ganhos (ou perdas) para a economia brasileira resultantes dos possíveis efeitos da agricultura de precisão.

Vale ressaltar, entretanto, que a presente análise se baseia em um modelo de funcionamento da economia brasileira que, como todos os modelos deste tipo, é aproximado, e não exato. Assim, as principais limitações desta análise são: assume retornos constantes à escala; os coeficientes técnicos são do ano de 2006; a oferta de recursos é infinita e elástica. 


\section{Resultados e discussão}

Para alcançar os resultados esperados neste trabalho, é apresentada uma análise sobre as condições da agricultura brasileira e do potencial de adoção da técnica de agricultura de precisão em 4.1. Neste passo, foram também identificados os produtos agrícolas utilizados na simulação de impactos e elaborados cenários para mensurar os possíveis impactos na economia brasileira resultantes da adoção da agricultura de precisão. Este último resultado foi apresentado no item 4.2.

\subsection{Produção agropecuária brasileira e elaboração dos cenários}

Conforme descrito na revisão feita anteriormente, o potencial de uso da agricultura de precisão para as condições brasileiras são as extensas áreas de lavoura de maneira a se ter variabilidade e que esta lavoura seja lucrativa para o produtor fazer uso da técnica. Além disto, devem ser analisados os produtos e insumos contemplados nos estudos revisados. Para encontrar este potencial, neste item foram utilizadas informações agropecuárias mais recentes para o Brasil, do censo de 2006 (IBGE, 2006).

A produção agropecuária neste censo está separada em 9 categorias: lavoura temporária; horticultura e floricultura; lavoura permanente; sementes, mudas e outras formas de propagação vegetal; pecuária e criação de outros animais; produção florestal - plantadas; produção florestal - nativas; pesca e aquicultura. Uma vez que a agricultura de precisão oferece benefícios ao produtor quando utilizada em áreas mais extensas, segundo IBGE (2006), das categorias descritas tem-se que a pecuária ocupa maior área $(62 \%$ do total com área média das propriedades de 90 ha), seguida das lavouras temporárias ( $25 \%$ do total e área média das propriedades de $44 \mathrm{ha}$ ) e das lavouras permanentes (6\% da área agrícola total e área média das propriedades de 34 ha). Entretanto, conforme observado nas Tabelas 1 e 2, os estudos de avaliação das 
técnicas de agricultura de precisão contemplaram apenas a produção agrícola de lavouras.

Assim, considerando as lavouras temporárias, dados do IBGE (2006) mostram que $63 \%$ do valor da produção destas lavouras ocorreu em lavouras com mais de 100 ha. Já para as lavouras permanentes, este percentual caiu para $42 \%$. Em termos de área plantada, $53 \%$ das lavouras temporárias estão em áreas com mais de 100 ha e 48\% das lavouras permanentes se encontram em áreas de mais de 100 ha. $\mathrm{O}$ tamanho de 100 ha adotado para esta descrição é considerado de grande extensão para uma lavoura e, portanto, com boas possibilidades de variabilidade no solo e, consequentemente, com condições favoráveis para obtenção de maior lucro pelo uso das técnicas de agricultura de precisão do que com a aplicação uniforme de insumos.

Entretanto, considerar o total de lavouras temporárias ou permanentes na análise pode acarretar em erros, uma vez que cada cultura pode ter características muito diferenciadas uma da outra. Assim, entre as lavouras, foram selecionadas para análise neste estudo aquelas que mais se destacaram no censo de 2006, tendo sido escolhidas a cana-deaçúcar (27\% do valor da produção e $11 \%$ da área plantada com lavouras temporárias), o milho (16\% e 24\%, respectivamente do valor da produção e da área plantada com lavouras temporárias) e a soja (24\% do valor da produção e $32 \%$ da área plantada com lavouras temporárias). Estes três produtos corresponderam a $67 \%$ do valor da produção e da área com lavoura temporária no país para o ano de 2006.

Uma vez que apenas as lavouras com área igual ou maior do que 100 ha foram selecionadas para identificação dos impactos da agricultura de precisão, foi necessário estimar os valores dos produtos na matriz insumo, considerando a produção apenas para estas lavouras. Para isto, com base nos dados da Produção Agrícola Municipal - PAM (IBGE, 2012), foram calculados o preço e a produtividade de cada um destes produtos. Uma vez que a matriz insumo-produto utilizada se refere ao ano de 2006, foram utilizadas as informações da produção deste mesmo 
ano. Multiplicadas estas variáveis pela área total colhida em propriedades com mais de 100 ha, tem-se o valor de produção equivalente para estas áreas. A Tabela 5 descreve estes dados.

Tabela 5. Área cultivada em lavouras com mais de 100 hectares, produtividade e preço médio da produção total das culturas em 2006 e valor da produção estimada para as lavouras com mais de 100 hectares

\begin{tabular}{lcccc}
\hline & $\begin{array}{c}\text { Área cultivada de } \\
\text { lavouras acima de } \\
\mathbf{1 0 0} \text { ha (ha) }\end{array}$ & $\begin{array}{c}\text { Produtividade } \\
\text { (Toneladas/ha) }\end{array}$ & $\begin{array}{c}\text { Preço } \\
\text { (R\$/tonelada) }\end{array}$ & $\begin{array}{c}\text { Valor da } \\
\text { produção } \\
\text { (milhões reais) }\end{array}$ \\
\hline Cana-de-açúcar & 4.869 .628 & 68,88 & 51,30 & 17.205 \\
Milho & 5.238 .062 & 3,61 & 268,74 & 5.076 \\
Soja & 12.360 .649 & 2,60 & 421,04 & 13.541 \\
\hline
\end{tabular}

Além disto, conforme descrito no item 3, os choques inseridos na MIP são relativos aos produtos finais consumidos na economia. Como os produtos analisados para uso da agricultura de precisão são demandados não apenas na sua forma bruta, mas também como processados, informações adicionais deste processo foram requeridas para estimar os choques na MIP. Para esta transformação, foram utilizadas informações da própria MIP, considerando apenas o processamento principal para cada produto: açúcar e etanol para a cana-de-açúcar; ração animal para o milho; e beneficiamento de óleo e farelo para a soja. Os dados utilizados são descritos na Tabela 6 . 
Tabela 6. Valores, em Milhões de Reais, da transformação de R\$ 1 milhão do produto bruto nos processados correspondentes e percentual do produto bruto processado

\begin{tabular}{|c|c|c|c|c|c|}
\hline Produto bruto & Unidade & Etanol & Açúcar & Ração & Óleo e farelo de soja \\
\hline Cana-de-açúcar & \multirow{3}{*}{$\begin{array}{l}\text { Milhões } \\
\text { de Reais }\end{array}$} & 1,34 & 2,26 & & \\
\hline Milho & & - & - & 2,61 & - \\
\hline Soja & & - & - & - & 3,05 \\
\hline $\begin{array}{l}\text { \% do produto bruto } \\
\text { transformado }\end{array}$ & $\%$ & $50 \%$ & $50 \%$ & $30 \%$ & $46 \%$ \\
\hline
\end{tabular}

Desta maneira, os valores da variável $Y_{l}$ (cenário base), sobre o qual os choques provenientes do uso da agricultura de precisão foram comparados, corresponderam a $\mathrm{R} \$ 3.564$ milhões para o milho $(70 \%$ do valor total); a R $\$ 7.273$ milhões para a soja (54\% do valor total); a $\mathrm{R} \$ 19.403$ milhões para o açúcar (utilizando $50 \%$ da cana); a $\mathrm{R} \$ 11.542$ milhões para o etanol (utilizando os demais $50 \%$ da cana); a $\mathrm{R} \$ 3.943$ milhões para a ração (utilizando os demais $30 \%$ do milho); e a $\mathrm{R} \$ 19.105$ milhões para óleo e farelo de soja (utilizando os demais $46 \%$ da soja).

Conforme também descrito no item 3, para analisar o impacto da agricultura de precisão, foi necessário alterar os coeficientes na MIP, os valores de aumento de produtividade, de redução e de aumento no consumo de adubos químicos. Neste aspecto, uma vez que os resultados encontrados na Tabela 1 não foram convergentes, foi simulado o valor de $10 \%$ de choque para todas as alterações descritas em cada um dos cenários estabelecidos no item anterior. Ou seja, foram simulados os seguintes cenários: (a) 10\% de aumento de produtividade; (b) 10\% de redução de consumo de fertilizantes; (c) $10 \%$ de aumento de produtividade com $10 \%$ de redução no uso de fertilizantes químicos; e (d) $10 \%$ de aumento de produtividade com $10 \%$ de aumento no uso de fertilizantes químicos. 


\subsection{Impactos na economia brasileira}

No item anterior, foram descritos os valores do choque de demanda para o potencial da agricultura analisada, que poderia ser impactada pela agricultura de precisão $\left(Y_{l}\right)$. A partir da matriz insumo-produto estimada para o ano de 2006 e da abertura da matriz para os setores agrícolas analisados (cana-de-açúcar, milho e soja), foram calculados as matrizes $A_{l}$ e os coeficientes emprego $\left(C_{E}\right)$, remuneração $\left(C_{R}\right)$, importação $\left(C_{M}\right)$ e PIB $\left(C_{P I B, I}\right)$.

Para mensurar os impactos resultantes do uso da agricultura de precisão na economia brasileira, conforme descrito no item 3 , foram então alteradas as matrizes descritas anteriormente de maneira a se obterem as matrizes $Y_{2}, A_{2}, A_{3}, A_{4}, A_{5}$ e as respectivas alterações nos valores do PIB $\left(C_{P I B, 2}\right.$, $C_{P I B, 3}, C_{P I B, 4}$ e $C_{P I B, 5}$ ).

Uma vez calculadas estas novas matrizes, foram então estimadas as equações descritas na Tabela 4 e, as Figuras 1 e 2 mostram os impactos totais (diretos, indiretos e de efeito renda) resultantes da diferença entre os resultados dos choques descritos nos cenários (a), (b), (c) e (d) e os resultados apresentados pelo choque do cenário base.

A Figura 1 mostra os impactos sobre produção, PIB, remuneração e importação (em milhões de reais) e a Figura 2, os impactos sobre o número de empregos. Observa-se que os resultados favoráveis para a economia brasileira, comparados ao cenário base (sem os benefícios estimados da agricultura de precisão), ocorreram apenas quando são observados ganhos na produtividade (cenários a, c e d) . No caso do cenário (a), por exemplo, os ganhos na economia foram da ordem de $\mathrm{R}$ \$ 20 bilhões de produção, R \$ 10,8 bilhões de PIB, R \$ 3 bilhões de remuneração e de cerca de 455 mil empregos. Observam-se, portanto, resultados positivos na economia, apesar do aumento nas importações ( $\mathrm{R} \$$ 0,6 bilhões). Os resultados apresentados nos cenários (c) e (d) tiveram valores muito próximos a estes resultados. Para se ter uma magnitude destes ganhos, o aumento do valor da produção na economia é de cerca de 
$75 \%$ do valor da produção de toda a cana-de-açúcar produzida no país em 2010 (IBGE, 2012). Entretanto, conforme pode ser observado na Figura 1, apenas cerca da metade destes impactos são advindos de impactos diretos e indiretos provocados pelo aumento de produtividade. Grande parte dos impactos são advindos do efeito renda que eles provocam na economia. Ou seja, com o aumento de emprego e renda provocado direta e indiretamente, as famílias aumentam seus gastos, incentivando outros setores da economia.

Assim, respondendo à questão apresentada neste estudo: sim, há crescimento econômico quando há lucro do produtor ao utilizar as técnicas de agricultura de precisão. Mas este impacto econômico significativo só ocorrerá se esta prática realmente aumentar a produtividade dos produtos agrícolas cultivados em grandes lavouras.

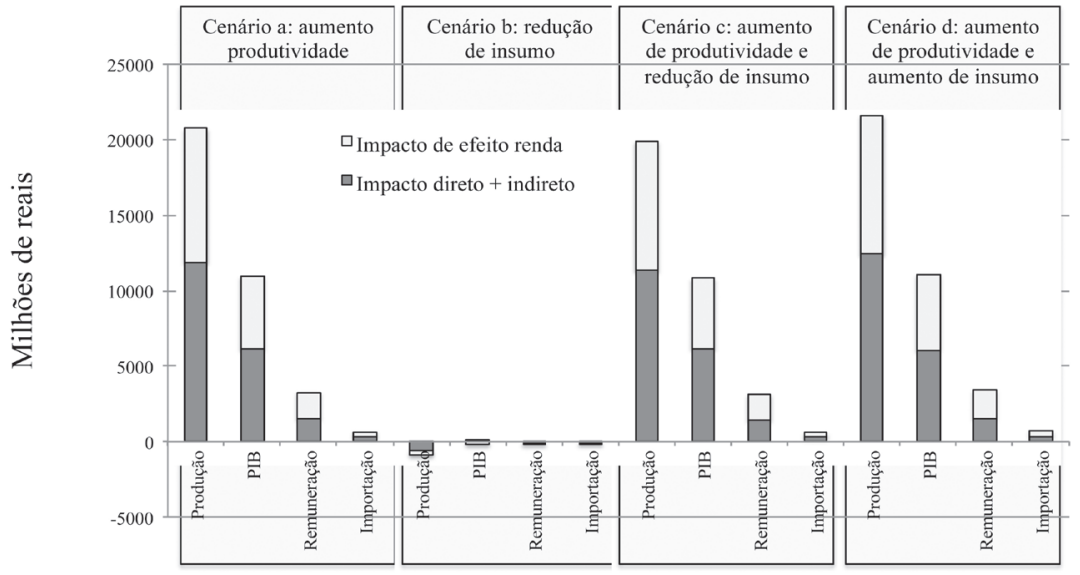

Figura 1. Impactos sobre a economia brasileira resultantes da diferença entre o cenário base e os cenários de aumento de produtividade (cenário a); de redução no uso de fertilizante (cenário b); de aumento de produtividade com redução de insumo (cenário c); e de aumento de produtividade com aumento de insumo (cenário d). 


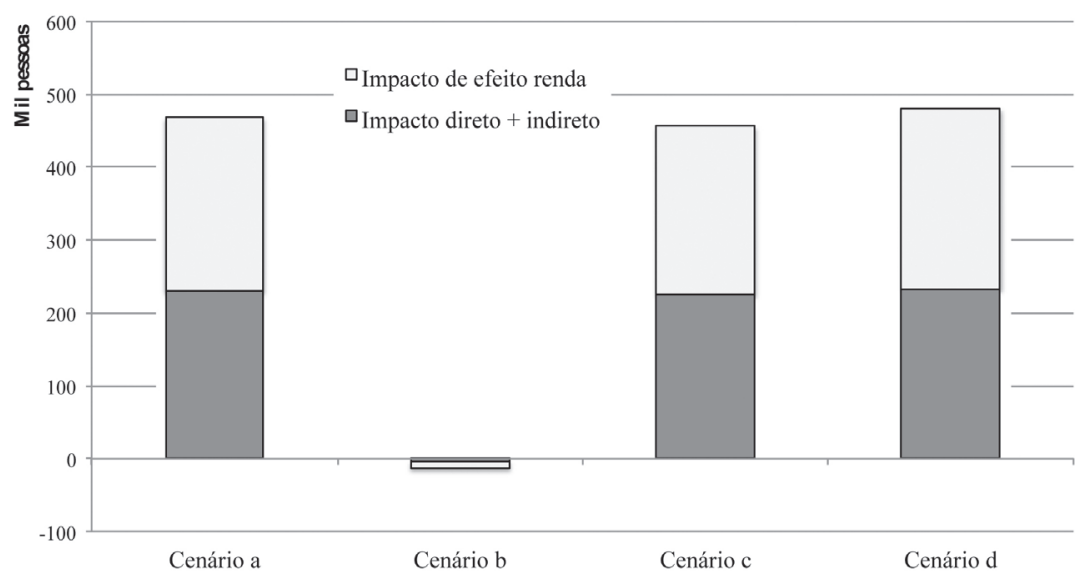

Figura 2. Impactos sobre o número de empregos gerados na economia brasileira resultantes da diferença entre o cenário base e os cenários de aumento de produtividade (cenário a); de redução no uso de fertilizante (cenário b); de aumento de produtividade com redução de insumo (cenário c); e de aumento de produtividade com aumento de insumo (cenário d).

Entretanto, se, ao invés do aumento de produtividade, ela for mantida constante, e os efeitos ocorrerem apenas por uma redução no uso dos insumos agropecuários, ou seja, se tivermos o cenário de redução no uso de fertilizantes (cenário b), os impactos estimados sobre toda economia, em relação ao cenário base, são desanimadores. Enquanto os resultados anteriores indicavam um impacto positivo na economia, estes últimos apresentam perdas da ordem de $\mathrm{R}$ \$ 0,9 bilhões de produção, de R $\$ 0,1$ bilhão de PIB, de R \$ 0,1 bilhão de remuneração e de cerca de menos 14 mil empregos. Apenas as importações deram impacto positivo, uma vez que foram reduzidas em $\mathrm{R} \$ 70$ milhões. Este resultado ocorreu porque os impactos positivos na economia originários do ganho de renda por parte dos produtores foram inferiores às perdas econômicas provocadas pela redução no consumo de fertilizantes, uma vez que apenas uma parte dos fertilizantes no país é importada, sendo grande parte ainda produzida no 
país. Assim, a redução de consumo deste insumo enfraquece a economia brasileira. Considerando a matriz econômica do ano de 2006 utilizada neste estudo, cerca de apenas $14 \%$ dos adubos e fertilizantes demandados foram importados. Contudo, como pode ser visualizado nas Figuras 1 e 2, estas perdas são muito pequenas quando comparadas aos ganhos obtidos nos demais cenários, podendo-se, inclusive, entendê-las como nulas, principalmente se houver maior quantidade de insumos importados. Assim, para este caso, a resposta à questão se os efeitos da agricultura de precisão podem gerar crescimento econômico é negativa.

Porém, a redução no uso de fertilizantes químicos tem um aspecto ambiental positivo, o que nos remete às informações dos impactos ambientais das técnicas de agricultura de precisão e a outra questão apresentada neste trabalho: não será o benefício da agricultura de precisão mais ambiental do que econômico? Entretanto, para responder a esta pergunta, precisamos, primeiramente, identificar corretamente os efeitos das técnicas de agricultura de precisão, ou seja, precisamos saber se eles são: (i) aumento de produtividade; (ii) redução no uso de insumos; ou apenas (iii) otimização dos insumos sem nenhum destes efeitos anteriores. Caso o efeito seja um dos dois últimos, a resposta pode ser sim. Isto porque, conforme os resultados apresentados para o cenário (b), mesmo reduzindo os custos do produtor e aumentando seus lucros (ii), os impactos na economia anulam tais ganhos. E, no último caso (iii), sem que ocorra mesmo o lucro para o produtor.

\section{Conclusões}

Estudos relacionados aos impactos sobre a produção agrícola do uso de técnicas de agricultura de precisão precisam ser revistos dada a falta de convergência dos resultados obtidos quanto à variação no uso de insumos e na produtividade. Além disto, para as condições nacionais, são necessários também estudos relacionados à redução na poluição ambiental provocada por estas técnicas. 
Este trabalho mostrou que se o benefício das técnicas de agricultura de precisão ocorrer sobre a produtividade agrícola, isto acarreta benefícios sociais (aumento de emprego) e econômicos (aumento de renda) para a economia brasileira. Entretanto, no caso de o benefício ser apenas de redução do insumo (fertilizantes no caso analisado), apesar de este causar aumento de lucro para o produtor rural, os benefícios para a economia como um todo não ocorrem, e a vantagem da técnica deve ser analisada apenas no sentido da redução de poluição.

Caso seja de interesse que as técnicas de agricultura de precisão contribuam para o crescimento econômico, seu estudo deve ser orientado para os efeitos sobre o aumento de produtividade.

\section{Referências}

ACOSTA, J.A.A.; BUSATO, M.R.; LONDERO, G.T.; LEMAINSKI, C.L.; SANTI, O.G.R. Uso de técnicas de agricultura de precisão no manejo da adubação do arroz irrigado. In: Congresso Brasileiro de Agricultura de Precisão - ConBAP, 2010. Ribeirão Preto. Anais...

AHMAD, S.; SUPALLA, R.J.; MILLER, W. Precision farming for profits and environmental quality: problems and opportunities. In: Annual Meeting of Agricultural Economics Association, Toronto, Canadá, 1997. Anais...

BARBIERI, D.M.; MARQUES JÚNIOR, J.; PEREIRA, G.T.; SANCHES, R.B.; PAZETO, R.J.; SIQUEIRA, D.S. Dependência espacial dos custos de fertilizantes para aplicação em taxa variada em cana-deaçúcar. In: Congresso Brasileiro de Agricultura de Precisão - ConBAP, 2008. Anais...

BONFIL, D.J.; MUFRADI, I.; ASIDO, S.; LONG, D.S. Precision nitrogen management based on nitrogen removal in rain fed wheat. In: $9^{\circ}$ International Conference on Precision Agriculture. July 20-23. Denver, Colorado. 2008. Anais... 
CARPENTIER, C.; BOSCH, D.; BATIE, S. Using spatial information to reduce costs of controlling agricultural nonpoint source pollution. Agricultural and Resource Economics Review, v. 27, n.1, pp. 72-84. 1998.

DELGADO, J.A.; BAUSCH, W.C. Potential use of precision conservation techniques to reduce nitrate leaching in irrigated crops. Journal of Soil Water Conservation, v. 60, n.6, p.379-387. 2005.

DELGADO, J.A.; BERRY, J.K. Advances in Precision Conservation. Advances in Agronomy, v.98, p.1-44. 2008.

DELGADO, J.A.; GROSS, C.M.; LAL, H.; COVER, H.; GAGLIARDI, P.; McKINNEY, S.P.; HESKETH, E.; SHAFFER, M.J. A New GIS nitrogen trading tool concept for conservation and reduction of reactive nitrogen losses to the environment. Advances in Agronomy, v. 105, p. 117-171. 2010.

DELGADO, J.A.; KHOSLA, R.; BAUSCH, W.C.; WESTFALL, D.G.; INMAN, D. Nitrogen fertilizer management based on site-specific management zones reduce potential for $\mathrm{NO}_{3}-\mathrm{N}$ leaching. Journal of Soil Water Conservation, v. 60, n.6, p. 402-410. 2005.

DU, Q.; CHANG, N.; YANG, C.; SRILAKSHMI, K.R. Combination of multispectral remote sensing, variable rate technology and environmental modeling for citrus pest management. Journal of Environmental Management, v. 86, n.1, p. 14-26. 2008.

ENGLISH, B.C.; ROBERTS, R.K.; LARSON, J.A. A logit analysis of precision farming tecnology adoption in Tennessee. Disponível em: http:// economics.ag.utk.edu/publications/precisionag/logit.pdf.

FAULIN, G.D.C.; MOLIN, J.P.; STANISLAVSKI, W.M. Influência da adubação em doses variáveis na produtividade da cultura do café (Coffea arabica L.) durante quatro safras consecutivas. In: Congresso Brasileiro de Agricultura de Precisão - ConBAP, 2010. Anais... 
GRIEPENTROG, H.W.; KYHN, M. Strategies for site specific fertilization in a highly productive agricultural region. In: $5^{\text {th }}$ International Conference on Precision Agriculture. 2000. Anais...

GUILHOTO, J.J.M., SESSO FILHO, U.A. Estimação da Matriz InsumoProduto a partir de dados preliminares das Contas Nacionais. Economia Aplicada, v.9, n.2, p. 277-299. 2005.

HEDLEY, C.B.; YULE, I.J. A high resolution soil water status mapping method for irrigation scheduling and two variable rate scenarios for pasture and maize irrigation. In: $9^{\circ}$ International Conference on Precision Agriculture. 20-23 julho. 2008. Anais...

HEISEL, T.; CHRISTENSEN, S.; WALTER, A. Weed managing model for patch spraying in cereal. In: Proceeding of the $3^{\text {rd }}$ International Conference on Precision Agriculture. ROBERT, P.C.; RUST, R.H.; LARSON, W.E. (Eds), p. 999-1007. 23-26 junho, 1996. Anais...

IBGE. Instituto Brasileiro de Geografia e Estatística. Censo Agropecuário. Disponível em: http://www.ibge.gov.br/home/estatistica/economia/ agropecuaria/censoagro/default.shtm. 2006.

IBGE. Instituto Brasileiro de Geografia e Estatística. Produção Agrícola Municipal. Disponível em: www.ibge.gov.br/home/estatistica/economia/ pam/2009. Acesso em: 01 de junho de 2012.

KOCH, B.; KHOSLA, R.; FRASIER, M.; WESTFALL, D.G. Economic feasibility of variable-rate nitrogen application in site specific management. Western Nutrient Management Conference, v.5, p. 107112. 2003. Anais...

LARSON, W.; LAMB, J.; KHAKURAL, B.; FERGUSON, R.; REHM, G. Potential of site-specific management for nonpoint environmental protection. In: PIERCE, F.; SADLER, E. (Eds). The state of site-specific management for agriculture.. Capítulo 15, p. 337-367. 1997. 
LONG, D.S.; ENGEL, R.E.; CARLSON, G.R. Method for precision nitrogen management in spring wheat: II. Implementation. Journal of Precision Agriculture, v. 2, n. 1, 25-38. 2000.

LOPES, F.A.; MOLIN, J.P. Adubação em doses variadas em citrus. In: Congresso Brasileiro de Agricultura de Precisão - ConBAP, 2010. Ribeirão Preto. Anais...

MCKINION, J.M.; JENKINS, J.N.; AKINS, D.; TURNER, S.B.; WILLERS, J.L.; JALLAS, E.; WHISLER, F.D. Analysis of a precision agriculture approach to cotton production. Computers and Eletronics in Agriculture, v. 32, n.3, p. 213-228. 2001.

MENEGATTI, L.A.A.; MOLIN, J.P.; GÓES, S.L.; KORNDORFER, G.H.; SOARES, R.A.B.; LIMA, E.A. Benefícios econômicos e agronômicos da adoção de agricultura de precisão em usinas de açúcar. In: $2^{\circ}$ Congresso Brasileiro de Agricultura de Precisão. São Pedro, SP. 2006. Anais...

MILLER, R.E.; BLAIR, P.D. Input-Output Analysis: Foundations and Extensions. Cambridge University Press: Cambrige. 2009.

MOLIN, J. P. . Agricultura de precisão. Parte 2: diagnóstico, aplicação localizada e considerações econômicas. Engenharia Agrícola, v. 17, n. 2, p. 108-121, 1997.

MOLIN, J.P.; MOTOMIYA, A.V.A.; FRASSON, F.R.; FAULIN, G.D.C.; TOSTA, W. Test procedure for variable rate fertilizer on coffee. Acta Scientiarum Agronomy, v. 32, n.4, p. 569-575. 2010.

MORTENSEN, D.; JOHNSON, G.; WYSE, D.; MARTIN, A. Managing spatially variable weed population. In: Proceeding of the $2^{\text {rd }}$ International Conference on Precision Agriculture. Madison, WI, EUA. 1994. Anais...

NORDMEYER, H.; HAUSLER, A.; NIEMANN, P. Patchy weed control as an approach in precision farming. In: $1^{\text {st }}$ European Conference on Precision Agriculture. Warwick, UK. 1997. Anais... 
PLANT, R.E. Site-specific management: the application of information technology to crop production. Journal of Computers and Electronics in Agriculture, v. 30, n.1 p. 9-29, 2001.

RAUN, W.R.; JOHNSON, G.V. Improving nitrogen use efficiency for cereal production. Agronomy Journal, v. 91, n. 3, p. 357-363. 1999.

ROBERTS, R.K.; ENGLISH, B.C.; MAHAMANESHETTI, S.B. Environmental and economic effects of spatial variability and weather. In: Proceedings of the $3^{\text {rd }}$ European Conference on Precision Agriculture. Montpellier. França. 2001. Anais...

ROBERTS, R.K.; MAHAMANASHETTI, S.B.; ENGLISH, B.C.; LARSON, J.A.; TYLER, D.D. Variable rate nitrogen application on corn fields: the role of special variability an weather. Journal of Agricultural and Applied Economics, v. 34, n.1, p.111-129. 2002.

RUFFO, M.L.; HENNINGER, A.S.; WIEBERS, M.; BELOW, F.E. Spatial variability of corn grain and ethanol responses to nitrogen fertilizer. In: $9^{\circ}$ International Conference on Precision Agriculture. July 20-23. Denver, Colorado. 2008. Anais...

SILVA, C.B.; MORAES, M.A.F.D.; MOLIN, J.P. Adoption and use of precision agriculture technologies in the sugarcane industry of São Paulo state, Brazil. Journal of Precision Agriculture, v. 12, n.1, p.67-81, 2011.

STAFFORD, J.; MILLER, P. Spatially variable treatments of weed patches. In: Proceeding of the $3^{\text {rd }}$ International Conference on Precision Agriculture. ROBERT, P.C.; RUST, R.H.; LARSON, W.E. (Eds), p. 465-474. 23-26 Junho, 1996. Anais...

STONE, M.L.; SOLIE, J.B.; RAUN, W.R.; TAYLOR, S.L.; RINGER, J.D.; WHITNEY, R.W. Use of spectral radiance for correcting in season fertilizer nitrogen deficiencies in winter wheat. Transactions of the ASABE, v. 39, n.5, p. 1623-1631.1996. 
TEKIN, A.B. Variable rate fertilizer application in Turkish wheat agriculture: economic assessment. African Journal of Agricultural Research, v.5, n. 8, p. 647-652. April, 2010.

TIAN, L.; REID, J.; HUMMEL, J. Development of a precision sprayer for site-specific weed management. Disponível em: http://age-web.age. uiuc.edu/faculty/lft/papers/sprayer.pdf. Acesso em: out. 2011. 2000.

TIMMERMANN, C.; GERHARDS, R.; KUHBAUCH, W. The economical impact of site-specific weed control. Journal of Precision Agriculture, v. 4, n. 3, p. 249-260. 2003.

TOZER, P.R.; ISBISTER, B.J. Is it economically feasible to harvest by management zone? Journal of Precision Agriculture, v. 8, n. 3, p. 151-159. 2007.

WEISZ, R.; FLEISCHER, S.; SMILOWITZ, Z. Site-specific integrated pest management for high value crops: Impact on potato pest management. Journal of Economic Entomology, v. 89, n. 2, p.501-509. 1996.

WHIPKER, L.D.; AKRIDGE, J.T. Precision Agricultural Services: Dealership Survey Results. Center for Food and Agricultural Business at Purdue University. Working Paper \#09-16. September 2009. 